\title{
A transient increase in total head phosphotyrosine levels is observed upon the emergence of Aedes aegypti from the pupal stage
}

\author{
Willy Jablonka, Raquel Senna, Thaisa Nahu, Guilherme Ventura, \\ Lidiane Menezes, Mário Alberto C Silva-Neto/+ \\ Programa de Biologia Molecular e Biotecnologia, Laboratório de Sinalização Celular, Instituto de Bioquímica Médica \\ e Instituto Nacional de Ciência e Tecnologia em Entomologia Molecular, Centro de Ciências da Saúde, \\ Universidade Federal do Rio de Janeiro, Av. Mal. Trompowski s/n, BI. D, Sala 5, 21949-902, Rio de Janeiro, RJ, Brasil
}

\begin{abstract}
Phosphorylation and dephosphorylation of protein tyrosine residues constitutes a major biochemical regulatory mechanism for the cell. We report a transient increase in the total tyrosine phosphorylation of the Aedes aegypti head during the first days after emergence from the pupal stage. This correlates with an initial reduction in total head protein tyrosine phosphatase (PTP) activity. Similarly, phosphotyrosine (pTyr)-containing bands are seen in extracts prepared from both male and female heads and are spread among a variety of structures including the antennae, proboscis and the maxillary palps combined with the proboscis. Also, mosquitoes treated with sodium orthovanadate, a classical PTP inhibitor, show reduced blood-feeding activity and higher head tyrosine phosphorylation levels. These results suggest that pTyr-mediated signalling pathways may play a role in the initial days following the emergence of the adult mosquito from the pupal stage.
\end{abstract}

Key words: Aedes aegypti - tyrosine phosphorylation - blood-feeding

Mosquito-borne diseases dramatically impact human populations. This is largely due to the absence of an effective public health vector control strategy (Arunachalam et al. 2010). Therefore, to provide efficient vector control, novel molecular tools are needed to inhibit the mosquito life cycle or impair its ability to feed on blood (van der Goes van Naters \& Carlson 2006). The mosquito life cycle is quite complex and is driven by a wide range of intracellular signalling events. Ongoing investigations have examined the mosquito reproductive signalling pathway (Attardo et al. 2005), but the major signalling pathways for other aspects of mosquito biology, such as blood-seeking behaviour, neuronal development and blood digestion remain unknown.

Phosphorylation and dephosphorylation of serine, threonine and tyrosine protein residues are powerful mechanisms that continuously drive cellular functions (Cohen 2002, Stoker 2005). These mechanisms often involve the concerted action of protein kinases, which catalyse protein phosphorylation, and protein phosphatases, which catalyse protein dephosphorylation. The actions of both enzyme families function to continuously tune a particular set of proteins in response to changes in cell environment. Currently, these enzymes have been the most promising source of targets for drug design in the XXI century (Cohen 2002, Stoker 2005). At present, pharmaceutical companies are taking great efforts to develop selective inhibitors to target a wide range of diseases (Cohen 2002).

\footnotetext{
Financial support: CNPq, FAPERJ, IFS, WHO, INCT

+ Corresponding author: maneto@bioqmed.ufrj.br

Received 29 November 2010

Accepted 14 July 2011
}

Phosphorylation of tyrosine residues induces phosphotyrosine (pTyr) signalling pathways. These are important signal transduction systems for many cellular functions such as protein synthesis and cell proliferation. The cellular ratio of pTyr to phosphoserine (pSer) and phosphothreonine (pThr) is 1:200:1800 (Wool et al. 1968, Schlessinger et al. 1983, Han et al. 2008), indicating that intracellular levels of pTyr are quite low but are localised to the initiating events of signalling pathways. These early events can play a regulatory role by mediating downstream phosphorylation events, which occur mainly on pSer- and pThr-containing proteins. pTyr levels are often decreased by protein tyrosine phosphatases (PTPs). These enzymes are divided into four families that are coded by 107 human genes. They are commonly involved in regulation of cellular immunity, metabolism (Alonso et al. 2004, Stoker 2005) and neuronal development, including the establishment of synapses, learning and memory (Stryker \& Johnson 2007). Many studies have addressed the role of protein tyrosine phosphorylation in the biology of blood-sucking insects (Hansen et al. 2005, Mesquita et al. 2005, Silveira et al. 2006, Riehle $\&$ Brown 2007). However, the dynamics of this process in the early life of adult mosquitoes has not been investigated.

The mosquito head is a complex region of the body that is composed of many different structures and tissues. It is responsible for vision, odour detection and feeding, as well as for the production of neurohormones related to reproduction and host-seeking behaviour (Predel et al. 2010). When Aedes aegypti males and females emerge from water they feed on sugar. After a period of 24-96 $\mathrm{h}$, the females will feed on blood for egg production. The blood-feeding behaviour turns Ae. aegypti mosquitoes into important vectors of arboviroses, such as yellow fever, dengue and West Nile encephalitis (Christophers 1960). 
When females are still not yet capable of blood-feeding, several molecular processes involving cuticle hardening and increased sensitivity to surrounding kairomones such as $\mathrm{CO}_{2}$ allows them to feed on blood (Sugumaran \& Semensi 1987, Grant \& O'Connell 2007). The molecular mechanisms that drive these processes are largely unknown. Despite the oscillation of juvenile hormone (JH) levels in the female hemolymph after emergence, allatectomised mosquitoes still exhibit host-seeking behaviour (Shapiro et al. 1986, Bowen \& Davis 1989). This suggests that an additional series of signalling events beyond those triggered by JH-coordinated mechanisms are involved in the generation of a blood-feeding mosquito in the days after emergence from the pupal stage.

In the present paper, we analyse protein tyrosine phosphorylation in the Ae. aegypti head on multiple days after emergence from the pupal stage and characterise the sodium orthovanadate (SO)-sensitivity of PTP from head extracts. We also show that $\mathrm{SO}$ treatment alters the female blood-feeding habits and head pTyr levels.

\section{MATERIALS AND METHODS}

Larvae and mosquitoes - Mosquitoes were maintained at $26-30^{\circ} \mathrm{C}$ and $60-80 \%$ humidity with a photoperiod of 12:12 h (light:dark). Larvae were reared in $20 \mathrm{x}$ $30 \times 5 \mathrm{~cm}$ pans with filtered water and fed with commercial dog food (Pedigree Junior, Masterfoods Brasil Alimentos Ltda, Mogi Mirim, SP, Brazil). Mosquitoes were fed ad libitum with $10 \%$ sucrose solutions and, whenever indicated, groups were fed a $10 \%$ sucrose solutions supplemented with $0.1 \mathrm{mM}$ of SO ad libitum.

Head homogenates and Western blotting - Fifteentwenty heads were collected at the same time between 01:30-02:00 pm in $1.5 \mathrm{~mL}$ polypropylene tubes on days one, three and six following mosquito emergence from the pupal stage and stored at $-20^{\circ} \mathrm{C}$. Heads were homogenised in cold $20 \mathrm{mM}$ Tris- $\mathrm{HCl}$ buffer $\mathrm{pH} 7.4$ in the presence of several protein phosphatase inhibitors (1 mM SO, $10 \mathrm{mM}$ sodium fluoride, $0.2 \mathrm{mg} / \mathrm{mL}$ sodium azide), 0.1 $\mathrm{mM}$ ethylenediamine tetraacetic acid (EDTA), $0.1 \mathrm{mM}$ ethylene glycol tetraacetic acid (EGTA), $1 \mathrm{mM}$ phenylmethylsulfonyl fluoride (PMSF), $0.1 \%$ Triton X-100 and $200 \mathrm{mM}$ sodium chloride. Western blots of different head parts were performed using heads from females six days after emergence. Proteins were extracted from separated antennae, proboscises with maxillary palps and heads without appendages in cold $20 \mathrm{mM}$ Tris- $\mathrm{HCl}$ buffer $\mathrm{pH}$ 7.4 also containing phosphatase inhibitors $(10 \mathrm{mM}$ sodium fluoride, $1 \mathrm{mM} \mathrm{SO}, 1 \mathrm{mM}$ phenylarsine oxide, 0.2 $\mathrm{mg} / \mathrm{mL}$ sodium azide), $1 \mathrm{mM}$ EDTA, $1 \mathrm{mM}$ EGTA, $0.1 \%$ Triton X-100 and $200 \mathrm{mM}$ sodium chloride in a 1:1 mixture with a protease inhibitor cocktail (P 8340 Sigma, Saint Louis, MO, USA) and $1 \mathrm{mM}$ PMSF. Homogenates were frozen at $-20^{\circ} \mathrm{C}$, thawed, sonicated for $10 \mathrm{~min}$ and centrifuged at $5,000 \mathrm{~g}$ at $4^{\circ} \mathrm{C}$ to eliminate cell debris. The supernatant was collected and centrifuged at $13,000 \mathrm{~g}$ at $4^{\circ} \mathrm{C}$ for $10 \mathrm{~min}$. This new supernatant was used for additional experiments. Protein concentration was determined by the Lowry method (Lowry et al. 1951) and bovine serum albumin (BSA) (Calbiochem, La Jolla, CA, USA) served as the protein standard. Forty micrograms of head proteins were separated on $0.75 \mathrm{~mm} 10 \%$ sodium dodecyl sulfate polyacrylamide gel electrophoresis gels (Laemmli 1970) and electroblotted onto polyvinylidene fluoride membranes (GE Healthcare) using a semidry system (Bio-Rad). Transferred proteins were visualised by Ponceau staining $(15 \% \mathrm{w} / \mathrm{v}$ tricholoroacetic acid and $0.36 \%$ $\mathrm{w} / \mathrm{v}$ Ponceau dye). After overnight blocking with 5\% BSA in Tris buffered saline-Tween (TBS-T) $(10 \mathrm{mM}$ Tris $\mathrm{pH}$ $8.0,150 \mathrm{mM}$ sodium chloride and $0.05 \%$ Tween-20), membranes were incubated with the primary anti-pTyr mouse monoclonal antibody (PY99) (Santa Cruz Biotechnology, Santa Cruz, CA, USA) at a 1:2,000 dilution in TBS-T for $2 \mathrm{~h}$ at room temperature. After three washes with TBS-T, membranes were incubated with an anti-mouse secondary antibody linked to horseradish peroxidase at a 1:10,000 dilution in TBS-T for $1 \mathrm{~h}$. After three washes with TBS (TBS-T without Tween-20), blots were developed in a chemiluminescence assay using the Enhanced Chemiluminescence System (GE Healthcare). Western blots were normalised to the Ponceau-stained membranes using the program ImageJ (Abramoff et al. 2004). Briefly, Western blotting films and Ponceau-stained membranes were scanned and the same areas were analysed in each lane by pixel density. This strategy provided a phosphate/protein ratio. To compare between days, a scale of arbitrary units was established where 1.0 was assigned to the phosphate/ protein ratio obtained for male heads on day one. This approach has been described in a previous study (Fialho et al. 2002). Additional details of the conditions are given in Silveira et al. (2006) or in Figure legends.

Biochemical characterisation of mosquito head PTP activity - PTP activity was characterised with female heads that were collected six days after emergence. Heads were homogenised in cold $20 \mathrm{mM}$ sodium acetate buffer $\mathrm{pH} 4.0$, frozen, thawed and centrifuged for $15 \mathrm{~min}$ at $13,140 \mathrm{~g}$ at $4^{\circ} \mathrm{C}$. The supernatant was collected and the protein concentration was determined by the Lowry method using BSA as the protein standard. The optimum $\mathrm{pH}$ for PTP activity was determined with the use of a mixture of buffers ( $0.1 \mathrm{M}$ sodium acetate, 0.1 $\mathrm{M}$ sodium citrate, $0.1 \mathrm{M}$ Tris and $0.1 \mathrm{M}$ glycine) and $\mathrm{pH}$ was adjusted between 3.0-8.0 with $\mathrm{HCl}$ or $\mathrm{NaOH}$. Assays were performed at $37^{\circ} \mathrm{C}$ for $60 \mathrm{~min}$, employing $0.5 \mathrm{mM}$ p-nitro-phenyl-phosphate (pNPP) as substrate and were terminated with $2 \mathrm{~N} \mathrm{NaOH}$ at a concentration of one part for every eight parts of the total assay volume. Where indicated, enzymatic assays of the time course of substrate hydrolysis, substrate concentration and presence of different phosphatase inhibitors were conducted as indicated in Figure legends $(1 \mu \mathrm{M}$ okadaic acid, $1 \mathrm{mM}$ sodium fluoride, $1 \mathrm{mM}$ phenylarsine oxide, $0.1 \mathrm{mM}$ ammonium molybdate, $0.1 \mathrm{mM} \mathrm{SO}, 0.1$ $\mathrm{mM}$ sodium pervanadate). The apparent molecular mass of the proteins responsible for the most significant PTP activity in head homogenates was determined by chromatography using a Superose 6 HR column coupled to a Shimadzu High Performance Liquid Chromatograph System equilibrated with $0.1 \mathrm{M}$ sodium acetate buffer pH 6.0 in $150 \mathrm{mM}$ sodium chloride. Eluates were examined using the same buffer and $0.5 \mathrm{~mL}$ samples were monitored by absorbance at $280 \mathrm{~nm}$. Fifty microliters of 
each fraction was assayed for PTP activity in the presence or absence of $0.1 \mathrm{mM} \mathrm{SO}$. The molecular mass of each fraction responsible for the PTP activity was estimated with the use of the following molecular mass standards eluted in the same buffer and conditions described above (ferritin: $442 \mathrm{kDa}$; catalase: $232 \mathrm{kDa}$; albumin: $69 \mathrm{kDa}$; ovalbumin: $47 \mathrm{kDa}$ ).

PTP activity following mosquito emergence - Mosquito heads were collected at the same time, between 01:30-02:00 pm on days one, three, and six following their emergence from the pupal stage. Head homogenates were prepared as described in the previous section. Three micrograms of head proteins were assayed in $20 \mathrm{mM}$ sodium acetate buffer $\mathrm{pH} 4.0$ at $37^{\circ} \mathrm{C}$ for 30 min using $0.5 \mathrm{mM}$ of pNPP and $0.1 \mathrm{mM} \mathrm{SO}$ served as the negative control. A more detailed description of the conditions may be found in Silveira et al. (2006).

SO treatment and blood-feeding test - Mosquitoes were pre-treated with a $10 \%$ sucrose solution or $10 \%$ sucrose solution supplemented with $0.1 \mathrm{mM} \mathrm{SO}$ for six days after emergence. During this time, death was not observed in either group. Before the blood-feeding test, mosquitoes were not starved. The blood-feeding test was carried out on the sixth day. In the absence of sucrose solution in the cage, rabbit ears were offered as a blood source for $10 \mathrm{~min}$. Blood-fed and unfed female mosquitoes were separated and counted in both groups. Western blot analysis of pTyr-containing proteins utilised 15-20 heads from female mosquitoes that underwent the same treatments described above and were collected between days 1-6 after emergence.

Statistical analysis - One-way analysis of variance (ANOVA) with Tukey's post hoc test and Student's paired t-test were performed using GraphPad Prism version 5.04 for Windows (GraphPad Software, La Jolla, CA, USA; graphpad.com)

Animal experimental procedures - Rabbits were used in accordance with the local committee for animal experimentation at the Health Sciences Centre, Federal University of Rio de Janeiro with the approval \# IBQM 011.

\section{RESULTS}

Tyrosine phosphorylation in mosquito heads increases after emergence - The transition from the pupal to adult stage imparts large changes on the mosquito life cycle. This transition to the terrestrial environment includes several molecular adaptations that are likely initiated by neuroendocrine modifications related to mosquito flight, vision, olfaction and feeding. Therefore, we asked whether the tyrosine phosphorylation profile of the mosquito head changes after emergence. As shown in Fig. 1A, male and female heads exhibit a similar banding pattern of tyrosine phosphorylated protein residues on all days investigated (a single band between 50-64 $\mathrm{kDa}, 2$ between 64-98 kDa and 1 above $98 \mathrm{kDa}$ ). Among these bands, two heavily phosphorylated protein bands (the band between $50-64 \mathrm{kDa}$ and the band above 98 $\mathrm{kDa}$ ) became more intense three days after emergence (Fig. 1A). The two bands between $64-98 \mathrm{kDa}$, however,
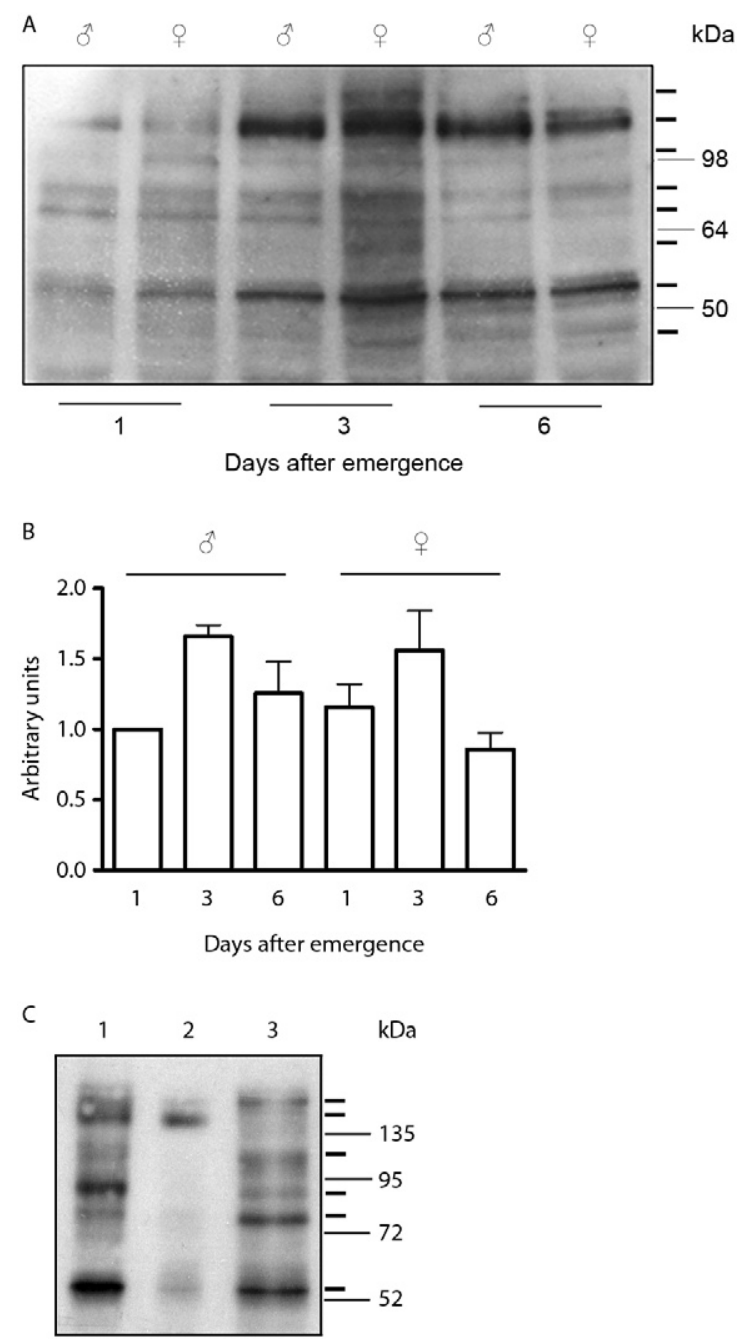

Fig. 1: mosquito heads undergo an increase of tyrosine phosphorylation levels after emergence from the pupal stage. A: mosquito head extracts obtained from both male and females on days one, three and six after emergence were evaluated by Western blotting using antiphosphotyrosine monoclonal antibody (PY99) (Santa Cruz Biotechnology, Santa Cruz, CA, USA). The blot shown is representative of at least three independent experiments; B: densitometric analysis of phosphate/protein ratios (arbitrary units) from six independent experiments are plotted using values for day one males as reference; C: tyrosine-phosphorylated bands are spread throughout the mosquito head. Different parts of female heads collected six days after emergence were dissected and extracts were evaluated by Western blotting using PY99. Lane 1: heads without the appendages; 2: proboscis and maxillary palps; 3: antennae.

became less intense six days after emergence (Fig. 1A). A faint band of nearly $98 \mathrm{kDa}$ was observed on each day except in males on the first day after emergence. Also, two bands (1 slightly below $64 \mathrm{kDa}$ and 1 slightly below $50 \mathrm{kDa}$ ) were observed only in female heads three days after emergence. Together, all bands from each lane demonstrate an overall transient increase in tyrosine phosphorylation from day one-three followed by an overall decrease in total phosphorylation six days after emergence of adults from the pupal stage (Fig. 1B). 
We also sought to analyse whether these phosphorylated bands were associated with different parts of the head. Female heads obtained on day six were carefully dissected into different parts and immunoblotting was repeated as in Fig. 1A. Fig. 1C reveals that each structure of the female mosquito head, the head without the appendages (Lane 1), the proboscis with maxillary palps (Lane 2) and the antennae (Lane 3), shows unique phosphorylation bands. Immunoblotting of mosquitoes six days after emergence reveals that preparations of female heads without the appendages (Fig. 1C, Lane 1) contain the four major bands seen in Fig. 1A (1 above $52 \mathrm{kDa}$, 2 between $72-95 \mathrm{kDa}$ and 1 above $135 \mathrm{kDa}$, as shown in Fig. 1C). Only two phosphorylated bands (1 above 52 $\mathrm{kDa}$ and a more intense band above $135 \mathrm{kDa}$ ) were detected in the proboscis (Fig. 1C, Lane 2). In Lane 3 of Fig. $1 \mathrm{C}$ we observed that preparations of the antennae carried at least five well-phosphorylated bands (1 above 52 $\mathrm{kDa}, 2$ between $72-95 \mathrm{kDa}, 1$ between $95-135 \mathrm{kDa}$ and 1 above $135 \mathrm{kDa}$ ). The bands in Fig. $1 \mathrm{C}$ were observed using a 10 -fold reduction in protein and a 10 -fold increase in exposure time compared to the conditions utilised in Fig. 1A. This strategy allowed us to detect phosphorylated bands from about 400 antennae. Because we used a peroxidase-conjugated secondary antibody to detect pTyr-containing proteins, it was possible that the immunoblot signals may be the result of residual peroxidase activity in the head. Immunoprecipitation with an anti-pTyr monoclonal antibodies revealed pTyr-containing proteins eliminating this concern (data not shown).

Together, these results show that both mosquito sexes share similar pTyr dynamics following moulting and that different parts of the female head contribute uniquely to the final profile when females are ready to feed on blood.

Biochemical characterisation of mosquito head PTP activity - The transient increase in the pTyr levels is attributed to a combination of the following events: (i) modulation of the expression of the target phosphoproteins, (ii) increased expression and activity of tyrosine kinases and (iii) decreased expression or activity of PTP. PTPs are easily assayed with the aid of the colorimetric substrate $\mathrm{pNPP}$. We utilised this assay to address the final possibility that transient changes in tyrosine phosphorylation are driven by modulation of PTP activity. Initially, we obtained general biochemical data regarding putative PTP activity in the head. As shown in Fig. 2, PTP activity in protein extracts of the head was linear up to $120 \mathrm{~min}$ (Fig. 2A) and approached saturation at 1.5 $\mathrm{mM}$ of the pNPP substrate (Fig. 2B). The enzyme activity was highest at pH 4.0 (Fig. 2C) and was inhibited by classical PTP inhibitors such as SO, sodium pervanadate and ammonium molybdate (Fig. 2D). The PTP activity was not inhibited by okadaic acid, a known inhibitor of Ser-Thr PP2A phosphatase (Fig. 2D). The putative molecular mass of the protein responsible for this activity was close to $100 \mathrm{kDa}$, which was reflected by a single peak of enzyme activity towards pNPP and was detected through gel filtration analysis of head extracts (Fig. 2E, F). These results show that mosquito head extracts contain significant PTP activity.
PTP activity is reduced after emergence - We next evaluated PTP activity in both male and female head extracts obtained in the first days after pupal emergence. Fig. 3 shows a decrease in PTP activity six days after emergence in both male and female heads and was significantly significant for the male samples. These data show that mosquito heads underwent a transient increase in pTyr levels that was likely to be partially mediated by a decrease in overall PTP activity.

SO treatment inhibited blood-feeding - After observing the transient change of pTyr levels in mosquito heads (Figs. 1A, B , 3) we were curious whether disruption of pTyr signalling would alter blood-feeding. To test this hypothesis we treated mosquitoes with $\mathrm{SO}$, a canonical PTP inhibitor, and offered rabbit ears as a blood source. The results shown in Table reveal that untreated groups of females demonstrated a high proportion of bloodfed to unfed individuals when given a feeding source. Treatment with SO altered this proportion significantly diminishing the percentage of blood-fed and increasing the percentage of unfed mosquitoes (Table).
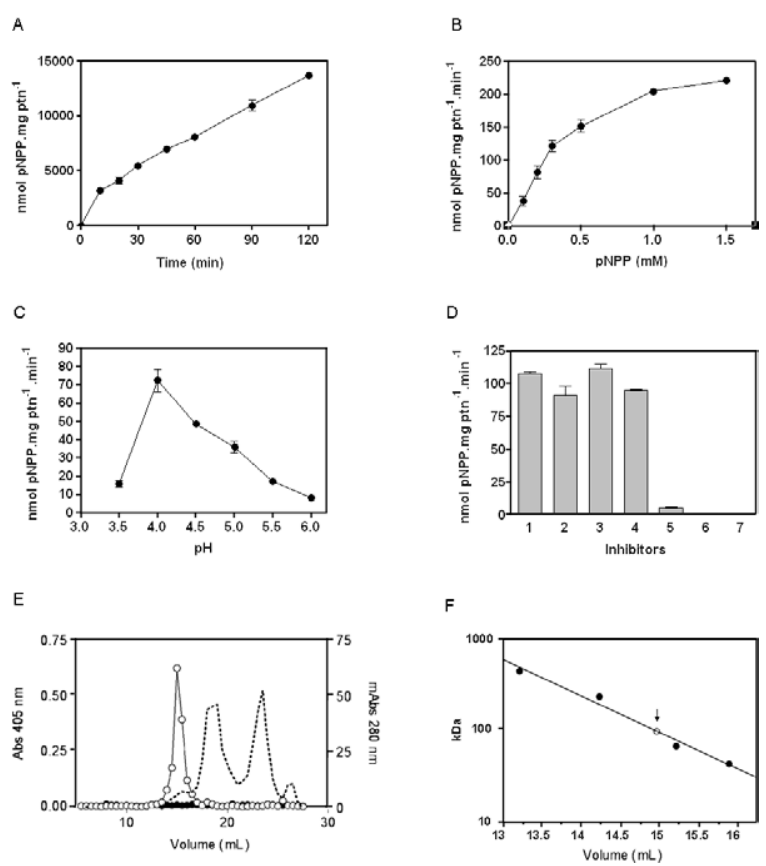

Fig. 2: enzymatic characterization of protein tyrosine phosphatase (PTP) activity in mosquito heads. A: time course of PTP activity with p-nitrophenyl-phosphate (pNPP) as substrate; B: substrate concentration curve; $\mathrm{C}: \mathrm{pH}$ curve; D: inhibitor screening (1: control; 2 : $10 \%$ dimethyl sulfoxide; 3: okadaic acid; 4: phenylarsine oxide; 5 : sodium orthovanadate (SO); 6: sodium pervanadate; 7: ammonium molybdate). Results are shown with mean and standard deviation from two independent experiments in triplicate; E: mosquito heads contain a major peak of PTP activity. Mosquito head extracts were chromatographed on a Superose 6HR column and fractions were assayed for PTP activity as described under Materials and Methods [open circles: total PTP activity; filled circles: PTP activity in the presence of SO; dotted line: $\mathrm{mAbs}$ at $280 \mathrm{~nm}$ (right axis)]; F: putative molecular mass associated with the PTP activity [filled circles: from left to right: ferritin (442 kDa), catalase (232 kDa), albumin (69 kDa), ovalbumin (47 $\mathrm{kDa}$ ); open circle: major peak of PTP activity from Fig. 2E]. 
SO treatment increased pTyr levels in mosquito heads - Fig. 4A, B show that females treated with SO also carried elevated levels of tyrosine-phosphorylated bands when heads were collected between one-six days after emergence. To more accurately monitor changes we reduced the exposure time of these Western blots compared with those in Fig. 1A. The immunoblot indicates that SO treatment may have inhibited tyrosine dephosphorylation in female heads by blocking PTP activity and that this modification may also participate in inhibiting blood-feeding as shown in Table.

\section{DISCUSSION}

We have characterised the tyrosine phosphorylation dynamics in the Ae. aegypti head after pupal emergence and have explored its contribution to the regulation of female blood-feeding behaviour. We observed a transient increase in total head protein tyrosine phosphorylation, which is common to both male and female heads (Fig. 1A, B). Two major phosphorylated tyrosine bands (1 between 50-64 kDa and 1 above $98 \mathrm{kDa}$ ) (Fig. 1A) were observed one, three and six days after mosquitoes emerged from the pupal stage. Because we employed a peroxidase-conjugated secondary antibody to detect pTyr-containing proteins, we considered the possibility that the immunoblot signals we detected were an artefact of residual peroxidase activity of the heads. This possibility was eliminated by immunoprecipitation with anti-pTyr monoclonal antibody, which also revealed pTyr-containing proteins (data not shown). This artefact was already detected by our group in immunblots containing insect tissues where peroxidases are commonly abundant.

We have also mapped the tyrosine phosphorylation profile of the head by analysing several head appendages (Fig. 1C). Interestingly, we found that the proboscis and maxillary palps showed just two bands of phosphorylated tyrosine (Fig. 1C, Lane 2). Mosquito maxillary palps and

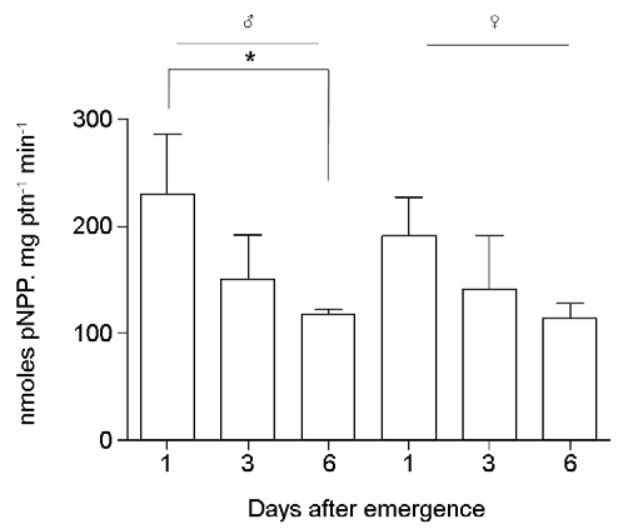

Fig. 3: protein tyrosine phosphatase (PTP) activity in mosquito heads decreases after emergence from the pupal stage. Both female and male mosquito heads were collected on different days (1, 3 and 6) after emergence and PTP activity was assayed as described. pNPP: p-nitrophenyl-phosphate. Bars represent mean and standard error of the mean of at least three independent experiments Asterisk means $p$ $<0.05$, one-way analyses of variance and, Turkey's post hoc test).

proboscis harbour sensilla associated with olfactory and gustatory neurons (Day 2005, Ignell \& Hansson 2005). Thus, the two pTyr bands seen in Fig. 1C (Lane 2) may be involved in tyrosine-based signalling processes in these cells. However, technical reasons have prevented us from localising the cells or quantifying the expression level of these proteins in intact heads compared to heads lacking appendages. The presence of several tyrosinephosphorylated proteins in the antennae may indicate an independent set of tyrosine phosphorylation signalling events in neurons with different functions such as those responsible for perception of odours, wind and vapour (Kellogg 1970). The identity of these phosphorylated proteins remains to be elucidated.

TABLE

Sodium orthovanadate (SO) treatment inhibits mosquito blood-feeding

\begin{tabular}{|c|c|c|c|c|c|c|}
\hline \multirow[b]{4}{*}{ Trial } & \multicolumn{6}{|c|}{ Treatment } \\
\hline & \multicolumn{3}{|c|}{ Sucrose } & \multicolumn{3}{|c|}{ Sucrose supplemented with SO } \\
\hline & \multicolumn{6}{|c|}{ Female blood-feeding test results on rabbit ears } \\
\hline & $\begin{array}{c}\mathrm{BF} \\
\mathrm{n}(\%)\end{array}$ & $\begin{array}{c}\text { NF } \\
\mathrm{n}(\%)\end{array}$ & $\sum_{\text {females }}$ & $\begin{array}{c}\text { BF } \\
\mathrm{n}(\%)\end{array}$ & $\begin{array}{c}\text { NF } \\
\mathrm{n}(\%)\end{array}$ & $\sum_{\text {females }}$ \\
\hline 1 & 34 (89.5) & $4(10.5)$ & 38 & $19(57.6)$ & $14(42.4)$ & 33 \\
\hline 2 & $52(86.7)$ & $8(13.3)$ & 60 & $45(80.4)$ & $11(19.6)$ & 56 \\
\hline 3 & $52(70.3)$ & $22(29.7)$ & 74 & $18(56.3)$ & $14(43.8)$ & 32 \\
\hline 4 & $28(77.8)$ & $8(22.2)$ & 36 & $21(53.8)$ & $18(46.2)$ & 39 \\
\hline$\%$ mean $\pm \mathrm{SD}$ & $81.1 \pm 8.7$ & $18.9 \pm 8.7$ & 208 & $62 \pm 12.4^{a}$ & $38 \pm 12.4^{b}$ & 160 \\
\hline
\end{tabular}

$a: \mathrm{p}<0.05$ blood fed (BF) female sucrose group vs. BF female sucrose plus SO group; $b: \mathrm{p}<0.05$ non fed (NF) female sucrose group vs. NF female sucrose plus SO group (Student's paired t-test). Mosquitoes (1:1 females:males) were treated with 10\% sucrose solution supplemented or not with $0.1 \mathrm{mM} \mathrm{SO}$. Female mosquitoes were then tested for blood-feeding, as described in Materials and Methods section. SD: standard deviation. 
Tyrosine phosphorylation has been studied in other insects such as Bombyx mori where it plays a role in ecdysteroid production by prothoracicotropic hormone stimulation in prothoracic glands (Lin \& Gu 2011). Another interesting example of PTP function is found in the wasp, Toxoneuron nigriceps, which parasitises Heliothis virescens (Lepidoptera: Noctuidae) by injecting bracovirus TnBV-infected eggs into the host larvae. This injection impairs the development of the host larvae by altering the function of the prothoracic gland. The authors suggest that this mechanism is controlled by viralderived PTPs that protect the emerging parasite larvae against the host immune defences (Falabella et al. 2006). The participation of PTPs has also been proposed for the enhanced locomotor activity of Baculovirus-infected $B$. mori larvae (Kamita et al. 2005).

Here, we characterised the activity of a PTP in mosquito head extracts. Our study shows that the Ae. aegypti head displays PTP activity under acidic conditions and that this PTP activity is associated with an estimated molecular mass of $100 \mathrm{kDa}$. A significant decrease in PTP activity is seen in male mosquito heads after emergence from the pupal stage (Fig. 3). This decrease correlates with previously reported reductions in dopamine levels that are present in male and female mosquito heads after emergence from the pupal stage (Andersen et al. 2006). Female mosquitoes have lower levels of dopamine than males and the increase in dopamine after blood-feeding is suggested to play an important role in ovary development (Andersen et al. 2006). The relationship between dopamine levels and the increase in tyrosine phosphorylation in the head is interesting because other models such as rat neurons have shown that dopamine injection leads to an increase in the tyrosine phosphorylation of a 180-kDa protein (Girault et al. 1992). In our system, the increase in specific tyrosine-phosphorylated proteins could be related in part to dopamine produced by neurosecretory cells of the mosquito brain. Increased dopamine levels after the pupal stage may induce an increase in tyrosine phosphorylation in the mosquito head by a mechanism that is common to both males and females. This model is particularly attractive because dopamine is related to cuticle sclerotisation, production of JH in corpora allata in Ae. aegypti (Andersen et al. 2006) and decision-making in the mushroom-body neurons of Drosophila (Zhang et al. 2007).

In this study, we hypothesised that tyrosine phosphorylation is important for blood-feeding so we tested mosquitoes treated with SO. Our data show a significant reduction in blood-feeding and increase of unfed female mosquitoes compared with control groups (Table). We propose that the SO ingested with the sucrose was absorbed in the midgut and carried by the hemolymph to tissues throughout the body. In Fig. 4A, we observed that SO treatment increased the intensity tyrosine-phosphorylated bands from mosquito heads, which is consistent with SO-mediated inhibition of PTP activities in vivo. From our observations, we correlate blood-feeding with higher pTyr protein content in the head (Fig. 4A, Table). However, SO treatment may also modify tyrosine-phosphorylation signalling in other parts of the mosquito body

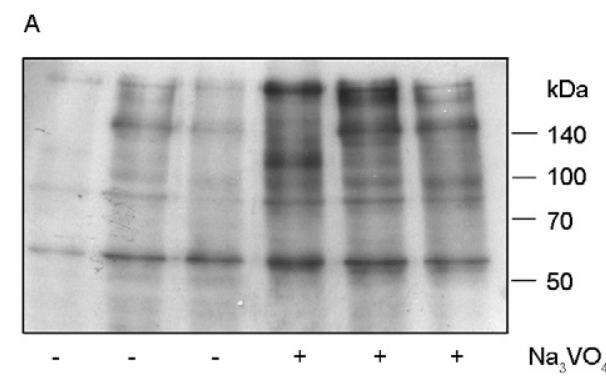

B

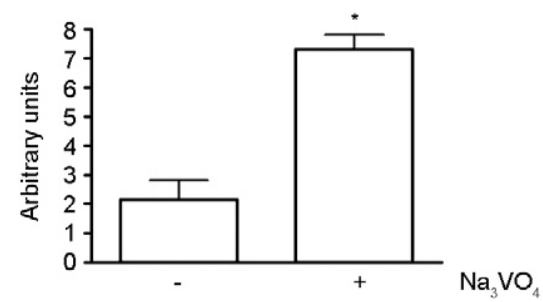

Fig. 4: presence of a protein tyrosine phosphatase inhibitor increases tyrosine phosphorylation in mosquito heads. A: immunoblotting with anti-phosphotyrosine antibody from heads of females treated with $10 \%$ sucrose (control) or $10 \%$ sucrose with $0.1 \mathrm{mM}$ sodium orthovanadate $\left(\mathrm{Na}_{3} \mathrm{VO}_{4}\right)$ collected between one-six days after emergence; $\mathrm{B}$ : densitometric analysis of panel A. Bars represent mean with standard deviation of the three independent experiments shown in A. Asterisk means $\mathrm{p}<0.05$ (Student's paired-t test).

and additional experiments must be performed to determine whether there is a specific molecular link between the increased levels of tyrosine-phosphorylated bands and the inhibition of blood-feeding that is observed with SO treatment (Fig. 4A, Table). We propose that tyrosinephosphorylation may enable mosquitoes to feed on blood. However, these processes are far more complex than the events reported here. It remains likely that a specific sequence of events combined with selective PTP inhibitors will eventually lead to a significant inhibition of bloodfeeding behaviour. Alternatively, it is possible that mosquitoes are more attracted to rabbit ears, and this may be confirmed in the future by conducting olfactometer experiments in SO-treated females.

In conclusion, our results are the first to demonstrate that Ae. aegypti tyrosine phosphorylation dynamics occur in mosquito heads after emergence from the pupal stage. The use of phosphoproteomic techniques followed by the identification of PTP genes in the mosquito genome, the sequencing of their target phosphoproteins and detailed behavioural studies will establish a close link between the events reported here. These data are important to understand basic mosquito head physiology and protein-signalling mechanisms. They may also contribute to the development of novel strategies to control mosquito blood-feeding behaviour and pathogen transmission.

\section{ACKNOWLEDGEMENTS}

To Katia Anastacio Laia, for technical assistance in mosquito rearing, to Dr Georgia Atella (IBqM-UFRJ), for help with the processing of images, and Dr Fernando Fernandez 
(Instituto de Biologia, UFRJ) and Dr Antonio Carlos de Freitas (Instituto de Biologia Roberto Alcântara Gomes, UERJ), for statistical consulting. This work is dedicated to Dr Martha Meriwether Sorenson who has reviewed with outstanding scientific criticism hundreds of manuscripts produced by the researchers of IBqM-UFRJ, Rio de Janeiro, Brazil in the last 27 years.

\section{REFERENCES}

Abramoff MD, Magelhaes PJ, Ram SJ 2004. Image processing with ImageJ. Biophotonics Int 11: 36-42.

Alonso A, Sasin J, Bottini N, Friedberg I, Osterman A, Godzik A, Hunter T, Dixon J, Mustelin T 2004. Protein tyrosine phosphatases in the human genome. Cell 117: 699-711.

Andersen JP, Schwartz A, Gramsbergen JB, Loeschcke V 2006. Dopamine levels in the mosquito Aedes aegypti during adult development, following blood feeding and in response to heat stress. J Insect Physiol 52: 1163-1170.

Arunachalam N, Tana S, Espino F, Kittayapong P, Abeyewickreme W, Wai KT, Tyagi BK, Kroeger A, Sommerfeld J, Petzold M 2010. Eco-bio-social determinants of dengue vector breeding: a multicountry study in urban and periurban Asia. Bull World Health Organ 88: 173-184.

Attardo GM, Hansen IA, Raikhel AS 2005. Nutritional regulation of vitellogenesis in mosquitoes: implications for anautogeny. Insect Biochem Mol Biol 35: 661-675.

Bowen MF, Davis EE 1989. The effects of allatectomy and juvenile hormone replacement on the development of host-seeking behaviour and lactic acid receptor sensitivity in the mosquito Aedes aegypti. Med Vet Entomol 3: 53-60.

Christophers SR 1960. Aedes aegypti (L.), the yellow fever mosquito: its life history, bionomics and structure, 1st ed., Cambridge University Press, Cambridge, 739 pp.

Cohen P 2002. Protein kinases - the major drug targets of the twentyfirst century? Nature Rev Drug Discov 1: 309-315.

Day JF 2005. Host seeking strategies of mosquito disease vectors. J Amer Mosquito Contr Assoc 21: 17-22.

Falabella P, Caccialupi P, Varricchio P, Malva C, Pennacchio F 2006. Protein tyrosine phosphatases of Toxoneuron nigriceps bracovirus as potential disrupters of host prothoracic gland function. Arch Insect Biochem Physiol 61: 157-169.

Fialho E, Silveira AB, Masuda H, Silva-Neto MA 2002. Oocyte fertilization triggers acid phosphatase activity during Rhodnius prolixus embryogenesis. Insect Biochem Mol Biol 32: 871-880.

Girault JA, Siciliano J, Robel L, Denisher V 1992. Stimulation of protein-tyrosine phosphorylation in rat striatum after lesion of dopamine neurons or chronic neuroleptic treatment. Proc Natl Acad Sci 89: 2769-2773.

Grant AJ, O'Connell RJ 2007. Age-related changes in female mosquito carbon dioxide detection. J Med Entomol 44: 617-623.

Han G, Ye M, Zou H 2008. Development of phosphopeptide enrichment techniques for phosphoproteome analysis. Analyst 133: 1128-1138.

Hansen IA, Attardo GM, Roy SG, Raikhel AS 2005. Target of rapamycin-dependent activation of S6 kinase is a central step in the transduction of nutritional signals during egg development in a mosquito. J Biol Chem 280: 20565-20572.

Ignell R, Hansson BS 2005. Projection patterns of gustatory neurons in the suboesophageal ganglion and tritocerebrum of mosquitoes. J Comp Neurol 492: 214-233.

Kamita SG, Nagasaka K, Chua JW, Shimada T, Mita K, Kobayashi M, Maeda S, Hammock BD 2005. A baculovirus-encoded protein tyrosine phosphatase gene induces enhanced locomotory activity in a lepidopteran host. Proc Natl Acad Sci USA 102: 2584-2589.

Kellogg FE 1970. Water vapor and carbon dioxide receptors in Aedes aegypti. J Insect Physiol 16: 99-108.

Laemmli UK 1970. Cleavage of structural proteins during the assembly of the head of bacteriophage T4. Nature 227: 680-685.

Lin JL, Gu SH 2011. Prothoracicotropic hormone induces tyrosine phosphorylation in prothoracic glands of the silkworm, Bombyx mori. Arch Insect Biochem Physiol 76: 144-155.

Lowry OH, Rosebrough NJ, Farr AL, Randall RJ 1951. Protein measurement with the folin-phenol reagent. J Biol Chem 193: 265-275.

Mesquita RD, Oliveira FBM, Shugar D, Fantappié MR, Silva-Neto MAC 2005. Nitrophorin synthesis is modulated by protein kinase CK2. Biochem Biophys Res Commun 335: 690-699.

Predel R, Neupert S, Garczynski SF, Crim JW, Brown MR, Russell WK, Kahnt J, Russell DH, Nachman RJ 2010. Neuropeptidomics of the mosquito Aedes aegypti. J Proteome Res 9: 2006-2015.

Riehle MA, Brown JM 2007. Characterization of phosphatase and tensin homolog expression in the mosquito Aedes aegypti: six splice variants with developmental tissue specificity. Insect Molec Biol 16: 277-286.

Schlessinger J, Schreiber AB, Levi A, Lax I, Libermann T, Yarden Y 1983. Regulation of cell proliferation by epidermal growth factor. CRC Crit Rev Biochem 14: 93-111.

Shapiro AB, Wheelock GD, Hagedorn HH, Baker FC, Tsai LW, Schooley DA 1986. Juvenile hormone and juvenile hormone esterase in adult females of the mosquito Aedes aegypti. J Insect Physiol 32: 867-877.

Silveira AB, Castro-Santos J, Senna R, Logullo C, Fialho E, SilvaNeto MAC 2006. Tick vitellin is dephosphorylated by a protein tyrosine phosphatase during egg development: effect of dephosphorylation on VT proteolysis. Insect Biochem Molec Biol 36: 200-209.

Stoker WA 2005. Protein tyrosine phosphatases and signaling. $J$ Endoc 185: 19-33.

Stryker E, Johnson KG 2007. LAR, liprin alpha and the regulation of active zone morphogenesis. J Cell Sci 120: 3723-3728.

Sugumaran M, Semensi V 1987. Sclerotization of mosquito cuticle. Experientia 43: 172-174.

van der Goes van Naters W, Carlson JR 2006. Insects as chemosensors of humans and crops. Nature 444: 302-307.

Wool IG, Stirewalt WS, Kurihara K, Low RB, Bailey P, Oyer D 1968. Mode of action of insulin in the regulation of protein biosynthesis in muscle. Recent Prog Horm Res 24: 139-213.

Zhang K, Guo JZ, Peng Y, Xi W, Guo A 2007. Dopamine-mushroom body circuit regulates saliency-based decision-making in Drosophila. Science 316: 1901-1904. 